\title{
Deep brain stimulation hardware-related infections: 10-year experience at a single institution
}

\author{
Kingsley O. Abode-lyamah, MD, ${ }^{1}$ Hsiu-Yin Chiang, PhD, ${ }^{2}$ Royce W. Woodroffe, MD, ${ }^{1}$ \\ Brian Park, MD, ${ }^{1}$ Francis J. Jareczek, BS, BA, ${ }^{3}$ Yasunori Nagahama, MD, ${ }^{1}$ Nolan Winslow, MD, ${ }^{3}$ \\ Loreen A. Herwaldt, MD, ${ }^{2,4,5}$ and Jeremy D. W. Greenlee, MD ${ }^{1}$ \\ Departments of ${ }^{1}$ Neurosurgery and ${ }^{2}$ Internal Medicine, ${ }^{3}$ University of lowa Carver College of Medicine; ${ }^{4}$ Program of Hospital \\ Epidemiology, University of lowa Hospitals and Clinics; and ${ }^{5}$ Department of Epidemiology, University of lowa College of Public \\ Health, lowa City, lowa
}

OBJECTIVE Deep brain stimulation is an effective surgical treatment for managing some neurological and psychiatric disorders. Infection related to the deep brain stimulator (DBS) hardware causes significant morbidity: hardware explantation may be required; initial disease symptoms such as tremor, rigidity, and bradykinesia may recur; and the medication requirements for adequate disease management may increase. These morbidities are of particular concern given that published DBS-related infection rates have been as high as $23 \%$. To date, however, the key risk factors for and the potential preventive measures against these infections remain largely uncharacterized. In this study, the authors endeavored to identify possible risk factors for DBS-related infection and analyze the efficacy of prophylactic intrawound vancomycin powder (VP).

METHODS The authors performed a retrospective cohort study of patients who had undergone primary DBS implantation at a single institution in the period from December 2005 through September 2015 to identify possible risk factors for surgical site infection (SSI) and to assess the impact of perioperative (before, during, and after surgery) prophylactic antibiotics on the SSI rate. They also evaluated the effect of a change in the National Healthcare Safety Network's definition of SSI on the number of infections detected. Statistical analyses were performed using the 2-sample t-test, the Wilcoxon rank-sum test, the chi-square test, Fisher's exact test, or logistic regression, as appropriate for the variables examined.

RESULTS Four hundred sixty-four electrodes were placed in 242 adults during 245 primary procedures over approximately 10.5 years; most patients underwent bilateral electrode implantation. Among the 245 procedures, 9 SSIs (3.7\%) occurred within 90 days and $16(6.5 \%)$ occurred within 1 year of DBS placement. Gram-positive bacteria were the most common etiological agents. Most patient- and procedure-related characteristics did not differ between those who had acquired an SSI and those who had not. The rate of SSIs among patients who had received intrawound VP was only $3.3 \%$ compared with $9.7 \%$ among those who had not received topical VP (OR $0.32,95 \% \mathrm{Cl} 0.10-1.02, \mathrm{p}=0.04)$. After controlling for patient sex, the association between VP and decreased SSI risk did not reach the predetermined level of significance (adjusted $\mathrm{OR} 0.32,95 \% \mathrm{Cl} 0.10-1.03, \mathrm{p}=0.06$ ). The $\mathrm{SSI}$ rates were similar after staged and unstaged implantations.

CONCLUSIONS While most patient-related and procedure-related factors assessed in this study were not associated with the risk for an SSI, the data did suggest that intrawound VP may help to reduce the SSI risk after DBS implantation. Furthermore, given the implications of SSI after DBS surgery and the frequency of infections occurring more than 90 days after implantation, continued follow-up for at least 1 year after such a procedure is prudent to establish the true burden of these infections and to properly treat them when they do occur.

https://thejns.org/doi/abs/10.3171/2017.9.JNS1780

KEY WORDS DBS complication; vancomycin powder; risk factors; prophylaxis; infection

ABBREVIATIONS ASA = American Society of Anesthesiologists; $\mathrm{BMI}=$ body mass index; $\mathrm{DBS}=$ deep brain stimulator; $\mathrm{GPI}=$ globus pallidus internus; $\mathrm{IPG}=$ implantable pulse generator; IV = intravenous; MSSA = methicillin-susceptible Staphylococcus aureus; NHSN = National Healthcare Safety Network; OCD = obsessive-compulsive disorder; SSI = surgical site infection; STN = subthalamic nucleus; VC = ventral capsule; VIM = ventralis intermedius; VP = vancomycin powder; VS = ventral striatum. SUBMITTED January 10, 2017. ACCEPTED September 25, 2017.

INCLUDE WHEN CITING Published online March 9, 2018; DOI: 10.3171/2017.9.JNS1780. 
$\mathrm{B}$ EKHTEREVA et al. were some of the first clinicians to employ deep brain stimulators (DBSs) in clinical practice in $1963 .{ }^{4}$ Since then, Benabid and colleagues' successful use of DBSs to treat Parkinson's disease in the $1980 \mathrm{~s}^{5,32}$ made this therapeutic device a regular part of clinical practice in the $1990 \mathrm{~s}$ and beyond. ${ }^{23}$ Subsequently, the DBS proved to be an effective surgical device for some movement ${ }^{15,18,30,44,45,52}$ and psychiatric disorders, ${ }^{26,33,34,36,38,39,48,51}$ and DBS implantation has become increasingly common, ${ }^{35}$ with estimates of more than 100,000 systems implanted world-wide. However, placement of these systems can be associated with significant complications, including stroke, death, and hardware-related infection.

Infections related to DBS systems are common, and patients may require additional hospitalizations and surgical procedures to eradicate such infections through complete or partial hardware removal and prolonged antibiotic therapy. ${ }^{7-10,16,19-21,25,28,41,42,46,50}$ Estimates of infection rates following DBS implantations differ but have been reported to be as high as $23 \%$. $^{7-10,16,20,21,25,28,41,42,46,50}$ Reported rates may vary in part because of discrepancies in the definition of surgical site infection (SSI), which can be affected by the duration of surveillance, whether reoperation is required, and whether superficial incisional infections are considered an SSI. ${ }^{7-10,16,20,21,25,28,41,42,46,50}$ The duration of surveillance is guided in part by the Centers for Disease Control and Prevention's National Healthcare Safety Network (NHSN), which in January 2013 decreased the duration of surveillance for SSI following hardware placement from 1 year to 90 days. $^{13,29}$

Despite the significance of such infections and the availability of various preventive measures, few studies have examined either risk factors for DBS-related infections or measures to prevent them. ${ }^{6,37}$ One recent beforeand-after study found that patients who used a preoperative $70 \%$ ethyl alcohol antiseptic skin wash had a lower SSI rate after DBS implantation than those who did not. ${ }^{28}$ Prophylactic antibiotics are recommended for DBS implantation; however, few studies have assessed whether specific antimicrobial agents are more effective than others. ${ }^{6}$ While some investigators have found that intrawound topical vancomycin powder (VP) is associated with lower SSI rates following spine operations ${ }^{14,31,40,47,49,53}$ and craniotomy, ${ }^{1}$ to our knowledge, only one study has assessed whether intrawound VP is associated with lower SSI rates after DBS placement procedures. ${ }^{43}$

To address this knowledge gap, we conducted a retrospective single-center cohort study to identify possible risk factors for DBS infections. We investigated the effect of patient characteristics and operative factors, including the number of procedures used to implant the DBS system, the antibiotic used for perioperative (before, during, and after surgery) prophylaxis, and the placement of intrawound VP, on SSI rates following DBS implantation.

\section{Methods \\ Study Design}

We conducted a retrospective cohort study of all adult patients ( $\geq 18$ years old) who had undergone primary
DBS implantation procedures performed by one surgeon (J.D.W.G.) between December 1, 2005, and September 30, 2015, at the University of Iowa Hospitals and Clinics (UIHC). Patients were identified using UIHC's electronic medical record. We reviewed each patient's record to obtain data on demographics, patient characteristics, procedural factors, and SSI. We included only those patients who had received DBS implants for the first time, regardless of whether the devices were implanted during a single "unstaged" procedure (concurrent implantation of lead[s], extension[s], and implantable pulse generator[s] [IPG]) or during staged procedures (lead[s] implanted during one procedure, and extension[s] and IPG[s] implanted during a subsequent procedure). We excluded patients who had undergone revision procedures or isolated IPG replacements. Data were collected to evaluate the incidence of SSI using both the current NHSN definition, which requires surveillance for only 90 days after implantation, and the prior NHSN definition, which required surveillance for 1 year after DBS implantation. ${ }^{13,29}$ One infection preventionist, who had more than 25 years' experience doing surveillance for SSI, independently identified the SSIs. Consistent with the NHSN definitions, we did not consider stitch abscesses or cellulitis to be an SSI. This study was approved by the University of Iowa Institutional Review Board.

\section{Surgical Technique}

The techniques for lead placement are the same for both staged and unstaged implantations. After a patient was deemed an appropriate candidate for DBS implantation, a movement disorder neurologist selected the appropriate target nucleus: typically the subthalamic nucleus (STN) or globus pallidus internus (GPI) for patients with Parkinson's disease, the ventralis intermedius (VIM) for patients with essential tremor, and the GPI for patients with dystonia. Indirect targeting was used for these patients, and intraoperative electrophysiology (microelectrode recording for GPI and STN targets and macrostimulation for VIM targets) was used to determine the final lead position. A small subset of patients, identified by psychiatric experts, underwent DBS implantation for medically refractory obsessive-compulsive disorder (OCD). For these patients, MRI-based direct targeting was used to place leads in the ventral capsule and ventral striatum (VC/VS). Most lead implantations were performed under local and monitored anesthesia; however, 3 patients required general anesthesia because they were very anxious. Microelectrode recording was not used for VC/VS placements.

Frame-based stereotaxy was used for all lead placements, and trajectories and entry points were chosen to avoid prominent sulci, the ventricles, and the caudate nucleus. Hair was clipped only around the areas to be incised, and 2-stage skin preps were done with either alcohol swabs or chlorhexidine/alcohol combination sponges (Chloraprep, BD Inc.) followed by povidone-iodine gel. Povidone-iodine adhesive skin drapes (Ioban, 3M) were applied.

All patients received prophylactic antibiotics intravenously 30-60 minutes before their incisions. Intracranial leads were placed under monitored anesthesia care. Standard stereotactic techniques were used for lead placement, 
and plastic caps (Stimloc, Medtronic Inc.) were used to secure the leads. For bilateral lead placements, one lead was tunneled subgaleally to the contralateral side of the head to facilitate placement of a single dual-channel IPG (for example, Activa PC, Medtronic Inc.). The distal lead ends were capped and coiled in a subgaleal or subperiosteal pocket around the burr hole cap. The wounds were irrigated copiously with a bacitracin and saline solution. In a subset of patients, approximately 200-250 mg of VP was placed in their incisions before wound closure.

\section{Single-Stage (Unstaged) Implantation}

For single-stage procedures, the stereotactic frame was removed after both frontal incisions had been closed. The patient was subsequently placed under general endotracheal anesthesia for implantation of the lead extensions and the IPG. The scalp, neck, and chest were prepared and draped. Incisions were made in the infraclavicular and retroauricular regions, and the frontal burr hole incision ipsilateral to the planned generator site was reopened. The DBS hardware was placed. In a subset of patients, approximately $400 \mathrm{mg}$ of VP was distributed among the frontal, retroauricular, and infraclavicular incisions before closure. All patients were admitted to the hospital after lead placements, and intravenous (IV) prophylactic antibiotics were continued for 24 hours postoperatively.

\section{Staged Implantation}

Most patients underwent 2-stage implantation procedures. The first procedure involved the placement of intracranial leads under monitored anesthesia care as described above. Postoperatively, patients were admitted to the hospital overnight and received IV prophylactic antibiotics for 24 hours. The second procedure typically occurred 7-10 days after lead implantation and was performed under general endotracheal anesthesia in an outpatient surgery setting. Each patient received prophylactic IV antibiotics prior to incision. In a subset of patients, VP was placed in the 3 incisions before wound closure during the secondstage procedure; thus, some patients received VP in their incisions during both procedures. Patients also received oral antibiotics for 24 hours after the second-stage procedure.

\section{Perioperative Antibiotics}

During the study period, we implemented several changes in perioperative antibiotic administration. From January 1, 2005, to May 31, 2011, all patients without penicillin allergies received perioperative IV nafcillin. On June 1, 2011, we began administering cefazolin perioperatively based on data from a study of neurosurgical spine procedures at our hospital ${ }^{27}$ and an update in clinical practice guidelines. ${ }^{12}$ Patients who were allergic to penicillin received either vancomycin or clindamycin, according to surgeon preference. Recent work in the surgical and neurosurgical literature has demonstrated the efficacy of topical vancomycin in preventing SSI. ${ }^{14,31,40,47,49,53}$ Starting March 1, 2012, all patients who were not allergic to vancomycin received VP in their surgical incisions; therefore, VP was not administered in a randomized fashion.

\section{Management of SSI}

Patients with SSI were managed using a lead-sparing algorithm initially described by Sillay et al. ${ }^{46}$ and modified based on experience at our institution. ${ }^{19}$ Briefly, localized infection at the site of the IPG or lead extensions was managed with explantation of the IPG and extensions. The distal ends of the preserved cranial leads were secured in antibiotic-impregnated ventricular catheter segments (approximately $4 \mathrm{~cm}$ long) containing clindamycin and rifampin (Bactiseal, Codman, Johnson \& Johnson). This was followed by a 6 - to 8 -week course of directed IV antibiotics based on intraoperative culture and antimicrobial sensitivities results; extensions and the IPG were reimplanted after resolution of the SSI and patients had been off all antibiotics for at least 6 weeks. Initial management of localized SSI involving a burr hole incision included incision and drainage followed by targeted IV antibiotics for 6-8 weeks. Our current practice is to treat any patients with gram-negative SSI, signs of meningitis, or widespread infection (that is, multiple scalp wounds involved) via complete hardware removal. All patients with SSI and preserved leads were followed up in the neurosurgery clinic for at least 6 months to ensure sustained resolution of infection.

\section{Statistical Analysis}

Statistical analysis was performed using SAS version 9.3 (SAS Institute Inc.). We used the 2-sample t-test for normally distributed continuous variables (for example, age, body mass index [BMI], blood glucose) or the Wilcoxon rank-sum test for continuous variables that were not normally distributed. For categorical variables (for example, sex, side of surgery, use of VP), we used the chi-square test or Fisher's exact test to compare proportions between non-SSI and SSI groups. The odds ratio and $\mathrm{p}$ value for each comparison were computed when appropriate. The $95 \%$ confidence intervals for the odds ratios, reflecting the range of possible odds ratios given the inherent variance of the data, were also determined. Given the independent association of patient sex with the SSI rate (see Results), we performed logistic regression to control for sex when determining the associations between VP use and SSI. All statistical tests were 2 -tailed. A p $<0.05$ was considered significant.

\section{Results}

During the 10.5-year study period, we identified 242 patients who had received 464 new electrode placements during 245 primary DBS implantation procedures. Nine SSIs $(3.7 \%)$ were detected within 90 days of DBS placement, and $16(6.5 \%)$ were detected within 1 year. The median time to the onset of all 16 SSIs was 79 days (range 30-244 days; Table 1); for the 9 SSIs that occurred fewer than 90 days after the procedure, the median time to onset was 50 days. Eleven infections (68.8\%) affected scalp wounds; 9 of these occurred at or near burr holes and 2 were associated with lead extensions. Five infections $(31.2 \%)$ affected chest wounds.

Given the change in our practice of using VP, we examined the SSI-causative pathogens since VP covers gram- 
TABLE 1. Infection occurrence and treatment

\begin{tabular}{|c|c|c|c|c|}
\hline SSI Organism & No. $(\%)$ & Days to SSI & Op Outcome & VP \\
\hline MSSA & $6(37.5)$ & & & \\
\hline \multirow[t]{4}{*}{ MSSA alone } & $4(25.0)$ & 51 & $\mathrm{LP} ;+$ & $\mathrm{N}$ \\
\hline & & 56 & NHR; + & $\mathrm{N}$ \\
\hline & & 97 & $\mathrm{LP} ;+$ & $\mathrm{N}$ \\
\hline & & 119 & $\mathrm{NHR} ;+$ & Y \\
\hline MSSA, Propionibacterium acnes & $1(6.3)$ & 156 & $\mathrm{LP} ;-$ & $\mathrm{N}$ \\
\hline MSSA, GPR suggestive of diphtheroids & $1(6.3)$ & 76 & NHR; + & $\mathrm{N}$ \\
\hline$P$. acnes & $7(43.8)^{*}$ & & & \\
\hline P. acnes alone & $1(6.3)$ & 32 & NHR; + & $\mathrm{Y}$ \\
\hline P. acnes, GPR suggestive of diphtheroids & $1(6.3)$ & 207 & $\mathrm{NHR} ;+$ & $\mathrm{N}$ \\
\hline P. acnes, Pseudomonas aeruginosa & $1(6.3)$ & 30 & LP; - & $\mathrm{N}$ \\
\hline P. acnes, methicillin-resistant CoNS, Staphylococcus epidermidis & $1(6.3)$ & 195 & NHR; - & $\mathrm{N}$ \\
\hline P. acnes, Enterobacter cloacae & $1(6.3)$ & 114 & LP; - & $\mathrm{N}$ \\
\hline P. acnes, S. lugdunensis & $1(6.3)$ & 244 & NHR; + & $\mathrm{N}$ \\
\hline Gram-positive cocci & $1(6.3)$ & 36 & $\mathrm{LP} ;+$ & $\mathrm{N}$ \\
\hline Methicillin-resistant S. epidermidis & $1(6.3)$ & 82 & NHR; + & Y \\
\hline Serratia marcescens & $1(6.3)$ & 38 & $\mathrm{~T} ;+$ & Y \\
\hline Mixed skin flora & $1(6.3)$ & 50 & NHR; + & $\mathrm{N}$ \\
\hline All infections & $16(100)$ & $30-244$ & & $4(25)$ \\
\hline
\end{tabular}

CoNS = coagulase-negative staphylococci; GPR = gram-positive rods; $L P=$ lead preservation technique (see also Dlouhy et al., 2012); N = no; NHR = no hardware removed; $T=$ total hardware removal; $Y=$ yes; + = hardware preserved; - = hardware ultimately removed.

* Includes 1 infection also reported with the MSSA SSI.

positive organisms. Gram-positive bacteria, including Propionibacterium acnes (7 cases [43.8\%]) and Staphylococcus aureus (6 cases [37.5\%]) alone or in combination with other organisms, were still the most common etiological agents (Table 1). Gram-negative organisms alone or in combination with $P$. acnes caused 3 SSIs $(18.8 \%)$. Five $(71.4 \%)$ of 7 patients with SSIs occurring after 90 days had polymicrobial infections, all of which included $P$. acnes. Among the 9 patients with SSIs occurring before 90 days, only 3 (33.3\%) had polymicrobial infections; this difference did not reach statistical significance. Any SSI from which P. acnes was isolated had a median onset of 156 days, compared with 56 days for SSIs caused by other organisms. However, the median time to SSIs caused by more-virulent organisms ( $S$. aureus, $S$. lugdunensis, and gram-negative organisms; 86.5 days) was longer than SSIs caused by less-virulent organisms (P.acnes, coagulase-negative staphylococci, and mixed skin flora; 66 days).

All 16 patients with SSI were readmitted, compared with only 8 patients $(3.5 \%)$ without SSI $(p<0.0001)$. Fifteen of the 16 patients with SSI were initially treated with hardware-preservation strategies, whereas 1 patient had upfront total hardware removal due to suspected SSI at multiple incision sites. Nine of the 16 patients had focal scalp SSI at or near the site of the burr hole. These SSIs were managed with incision and drainage without hardware explantation, followed by an extended course of antibiotics ("NHR" in Table 1); only 1 of these 9 patients later developed SSI relapse necessitating hardware removal. Six (37.5\%) of the 16 patients with SSI had infection at the site of the IPG or lead/extension junction, and this hardware was removed without cranial lead explantation per our lead-sparing algorithm ("LP" in Table 1)..$^{19}$ While all 6 of these patients underwent re-implantation of the IPG and extensions, 3 of the 6 had delayed SSI recurrence and ultimately required total hardware removal. All SSI patients were closely followed up to ensure resolution of infection.

Patients with SSI and those without were similar with respect to age, BMI, preoperative glucose level, American Society of Anesthesiologists (ASA) score, preoperative diagnosis, procedure laterality, procedure duration, and antibiotic used for perioperative IV antimicrobial prophylaxis (Table 2). Male patients were less likely than female patients to acquire an SSI (OR $0.28,95 \%$ CI $0.10-0.80, \mathrm{p}$ $=0.01$ ) after DBS implantation procedures. Although the IV antibiotic used for prophylaxis was not significantly associated with the incidence of infection, none of the 17 patients who had received vancomycin developed an SSI. Twelve $(9.7 \%)$ of the 124 patients who had not received intrawound VP had an SSI, whereas only $4(3.3 \%)$ of the 121 patients who had received topical VP had an SSI. Approximately half of the patients without SSI and $25.0 \%$ of those with SSI had received intraoperative topical VP (OR 0.32, 95\% CI 0.10-1.02, p = 0.04). After controlling for patient sex via logistic regression, the association between VP and decreased SSI risk did not quite reach the predefined level of significance (adjusted OR 0.32, 95\% CI $0.10-1.03, \mathrm{p}=0.06$ ). Wound dehiscence was observed in more patients with SSI than in those without $(25.0 \%$ vs $2.2 \%$, OR $14.9,95 \%$ CI 3.5-62.9, $\mathrm{p}<0.01)$. Wound dehiscence rates were similar for the VP and non-VP groups.

Overall, 17 procedures $(6.9 \%)$ were unstaged implanta- 
TABLE 2. Characteristics of patients and procedures stratified by the presence or absence of SSI

\begin{tabular}{|c|c|c|c|c|c|}
\hline Variable & All Patients & No SSI & SSI & OR for SSI (95\% CI) & $p$ Value \\
\hline No. of patients & 245 & 229 & 16 & & \\
\hline \multicolumn{6}{|l|}{ Patient-related factors } \\
\hline Mean age at surgery in yrs & $62.9 \pm 11.3$ & $62.7 \pm 11.5$ & $65.6 \pm 6.7$ & & 0.13 \\
\hline Male sex & $162(66.1)$ & $156(68.1)$ & $6(37.5)$ & $0.28(0.10-0.80)$ & 0.01 \\
\hline Mean BMl in kg/m² & $29.2 \pm 6.3$ & $29.2 \pm 6.4$ & $30.3 \pm 4.6$ & & 0.49 \\
\hline Mean blood glucose in $\mathrm{mg} / \mathrm{dl}^{*} \dagger$ & $104.9 \pm 34.1$ & $105 \pm 33.6$ & $104.1 \pm 40.9$ & & 0.92 \\
\hline ASA score $\geq 3 \dagger$ & $125(51.2)$ & $119(52.2)$ & $6(37.5)$ & $0.55(0.19-1.56)$ & 0.26 \\
\hline \multicolumn{6}{|l|}{ Preop diagnosis } \\
\hline Dystonia & $13(5.3)$ & $12(5.2)$ & $1(6.3)$ & $1.21(0.15-9.90)$ & 0.59 \\
\hline Essential tremor & $94(38.4)$ & $86(37.6)$ & $8(50.0)$ & $1.66(0.60-4.59)$ & 0.32 \\
\hline OCD & $6(2.4)$ & $6(2.6)$ & $0(0)$ & & 1.00 \\
\hline Parkinson's disease & $131(53.5)$ & $124(54.1)$ & $7(43.8)$ & $0.66(0.24-1.83)$ & 0.42 \\
\hline Post-stroke thalamic pain syndrome & $1(0.4)$ & $1(0.4)$ & $0(0)$ & & 1.00 \\
\hline \multicolumn{6}{|l|}{ Procedure-related factors } \\
\hline \multicolumn{6}{|l|}{ Side of surgery } \\
\hline Bilat & $219(89.4)$ & $204(89.1)$ & $15(93.8)$ & $1.84(0.23-14.5)$ & 1.00 \\
\hline $\mathrm{Lt}$ & $12(4.9)$ & $12(5.2)$ & $0(0)$ & & 1.00 \\
\hline Rt & $14(5.7)$ & $13(5.7)$ & $1(6.3)$ & $1.11(0.14-9.09)$ & 1.00 \\
\hline \multicolumn{6}{|l|}{ Anatomical target } \\
\hline GPI & $48(19.6)$ & $45(19.7)$ & $3(18.8)$ & $0.94(0.26-3.45)$ & 1.00 \\
\hline STN & $98(40.0)$ & $93(40.6)$ & $5(31.3)$ & $0.66(0.22-1.98)$ & 0.46 \\
\hline VC/VS & $6(2.4)$ & $6(2.6)$ & $0(0)$ & & 1.00 \\
\hline VIM & $93(38.0)$ & $85(37.1)$ & $8(50.0)$ & $1.69(0.61-4.68)$ & 0.30 \\
\hline Staged procedure & $228(93.1)$ & $213(93.0)$ & $15(93.8)$ & $1.13(0.14-9.08)$ & 1.00 \\
\hline Median time to 2 nd stage in days (range) $\ddagger$ & $10(0-346)$ & $10(0-84)$ & $9(7-346)$ & & 0.61 \\
\hline Mean op duration in minst§ & $168.3 \pm 46.9$ & $167.6 \pm 47.0$ & $178.3 \pm 44.5$ & & 0.38 \\
\hline \multicolumn{6}{|l|}{ Other factors } \\
\hline \multicolumn{6}{|l|}{ Antimicrobial prophylaxis } \\
\hline Cefazolin & $151(61.6)$ & $140(61.1)$ & $11(68.8)$ & $1.40(0.47-4.16)$ & 0.54 \\
\hline Nafcillin & $52(21.2)$ & $48(21.0)$ & $3(18.8)$ & $0.87(0.24-3.18)$ & 1.00 \\
\hline Vancomycin & $17(6.9)$ & $17(7.4)$ & $0(0)$ & & 0.61 \\
\hline Other & $26(10.6)$ & $24(10.5)$ & $2(12.5)$ & $1.22(0.26-5.70)$ & 0.68 \\
\hline Topical VP & $121(49.4)$ & $117(51.1)$ & $4(25.0)$ & $0.32(0.10-1.02)$ & 0.04 \\
\hline Postop seroma & $1(0.4)$ & $0(0)$ & $1(6.3)$ & & 0.07 \\
\hline Wound dehiscence & $9(3.7)$ & $5(2.2)$ & $4(25.0)$ & $14.9(3.5-62.9)$ & $<0.01$ \\
\hline
\end{tabular}

Values expressed as the mean \pm standard deviation or as number (\%), unless indicated otherwise. Boldface type indicates statistical significance.

* Blood glucose was recorded during a preoperative assessment within the month preceding the procedure.

† Available data: All Patients, 244; No SSI, 228.

$\ddagger$ Available data: All Patients, 228; No SSI, 213; SSI, 15

$\S$ For staged procedures, overall duration was defined as the sum of time from incision to wound closure for each stage.

tions, only $1(5.9 \%)$ of which was bilateral; 228 procedures $(93.1 \%)$ were staged, and $218(95.6 \%)$ of these were bilateral (Table 3). Patients with Parkinson's disease (OR 3.54, 95\% CI 1.43-8.77, $\mathrm{p}=0.004)$ and those with ASA scores $\geq 3$ (OR 3.77, 95\% CI 1.45-9.80, $\mathrm{p}=0.004)$ were more likely to undergo bilateral procedures rather than unilateral procedures. In addition, these patients were more likely to undergo staged rather than unstaged procedures. Given their underlying diseases, patients who underwent staged procedures were probably sicker than those who underwent unstaged DBS implantation. Nonetheless, the
SSI rate was similar between patients who underwent staged (6.6\%) and those who underwent unstaged (5.6\%) procedures. Rates of other complications, such as wound dehiscence and postoperative seroma, did not differ between the staged and unstaged groups.

\section{Discussion}

Our retrospective cohort study is the first to compare the SSI rate after DBS implantation between patients who received VP and those who did not. We found that intra- 
TABLE 3. Characteristics of patients and procedures stratified by staged and unstaged implantation

\begin{tabular}{|c|c|c|c|c|}
\hline Variable & Staged Procedure & Unstaged Procedure & OR for Staged Procedure $(95 \% \mathrm{Cl})$ & $\mathrm{p}$ Value \\
\hline No. of procedures & 228 & 17 & & \\
\hline \multicolumn{5}{|l|}{ Patient-related factors } \\
\hline Mean age at surgery in yrs & $63.0 \pm 11.0$ & $60.8 \pm 14.2$ & & 0.43 \\
\hline Male sex & $150(65.8)$ & $12(70.6)$ & $0.80(0.27-2.36)$ & 0.69 \\
\hline Mean BMl in $\mathrm{kg} / \mathrm{m}^{2}$ & $29.3 \pm 6.4$ & $28.6 \pm 4.8$ & & 0.70 \\
\hline Mean blood glucose in mg/dl ${ }^{*}$ & $105.6 \pm 34.9$ & $95.6 \pm 18.0$ & & 0.05 \\
\hline ASA score $\geq 3 \dagger$ & $121(53.1)$ & $4(25.0)$ & $3.39(1.06-10.83)$ & 0.03 \\
\hline \multicolumn{5}{|l|}{ Diagnosis } \\
\hline Dystonia & $13(5.7)$ & $0(0)$ & & 0.61 \\
\hline Essential tremor & $83(36.4)$ & $11(64.7)$ & $0.31(0.11-0.88)$ & 0.02 \\
\hline OCD & $5(2.2)$ & $1(5.9)$ & $0.36(0.04-3.26)$ & 0.35 \\
\hline Parkinson's disease & $127(55.7)$ & $4(23.5)$ & $4.09(1.29-12.92)$ & 0.01 \\
\hline Post-stroke thalamic pain syndrome & $0(0)$ & $1(5.9)$ & & 0.07 \\
\hline \multicolumn{5}{|l|}{ Procedure-related factors } \\
\hline \multicolumn{5}{|l|}{ Side of surgery } \\
\hline Bilat & $218(95.6)$ & $1(5.9)$ & $348.8(42.0-2898.3)$ & $<0.01$ \\
\hline $\mathrm{Lt}$ & $5(2.2)$ & $7(41.2)$ & $0.03(0.01-0.12)$ & $<0.01$ \\
\hline Rt & $5(2.2)$ & $9(52.9)$ & $0.02(0.01-0.07)$ & $<0.01$ \\
\hline Mean op duration in minst‡ & $168.4 \pm 47.6$ & $167.0 \pm 35.9$ & & 0.91 \\
\hline \multicolumn{5}{|l|}{ Other factors } \\
\hline \multicolumn{5}{|l|}{ Antimicrobial prophylaxis } \\
\hline Cefazolin & $141(61.8)$ & $10(58.8)$ & $1.13(0.42-3.09)$ & 0.81 \\
\hline Nafcillin & $47(20.6)$ & $4(23.5)$ & $0.84(0.26-2.71)$ & 0.76 \\
\hline Vancomycin & $15(6.6)$ & $2(11.8)$ & $0.53(0.11-2.53)$ & 0.33 \\
\hline Other & $25(11.0)$ & $1(5.9)$ & $1.97(0.25-15.50)$ & 1.00 \\
\hline Topical VP & $113(49.6)$ & $8(47.1)$ & $1.11(0.41-2.97)$ & 0.84 \\
\hline Postop seroma & $1(0.4)$ & $0(0)$ & & 1.00 \\
\hline Wound dehiscence & $9(4.0)$ & $0(0)$ & & 1.00 \\
\hline SSI & $15(6.6)$ & $1(5.9)$ & $1.13(0.14-9.08)$ & 1.00 \\
\hline
\end{tabular}

wound topical VP was associated with a lower SSI rate following DBS implantation than the rate observed after our standard practice, suggesting that topical VP may be an important preventive measure for patients undergoing this procedure. In addition, we found that men were less likely than women to acquire an SSI and that $43.8 \%$ of the infections occurred more than 90 days after the procedures.

Intrawound VP provides supratherapeutic antibiotic concentrations locally ${ }^{3}$ while reducing the risk of toxicities seen with systemic delivery. ${ }^{22}$ Numerous groups have found that intrawound VP is associated with decreased SSI rates after spinal procedures, 1,3,14,24,31,40,47,49,53 and neurosurgeons are now using topical VP to prevent SSI after other procedures, including craniotomy and cranioplasty. ${ }^{1,2}$ To date, only one other group has described the use of VP among patients undergoing DBS implantation. ${ }^{43}$ Rasouli and Kopell reported a case series of 297 patients who had undergone DBS or IPG placement or replacement. All DBS placements were staged. Fifty percent of the patients underwent primary procedures, and all patients received $1000 \mathrm{mg}$ of intrawound VP. Patients who had undergone DBS placement received $1000 \mathrm{mg}$ of vancomycin every 12 hours and $1500 \mathrm{mg}$ of cefuroxime every 8 hours intravenously for the first 24 hours in the postoperative period. Patients who had undergone IPG placements received 100 $\mathrm{mg}$ of doxycycline by mouth every 12 hours during the first 24 hours of the postoperative period. Four (1.3\%) of the 297 patients, all of whom had Parkinson's disease, acquired SSIs. That SSI rate was lower than the $3.3 \%$ rate in our patients who had received intrawound VP. Given that $50 \%$ of their patients underwent reoperations and $72 \%$ underwent procedures for the treatment of Parkinson's disease, one would expect their SSI rate to be higher than that in our patients, who all underwent primary procedures and only $53.5 \%$ of whom had Parkinson's disease. However, Rasouli and Kopell used a higher dose of intrawound VP (1000 mg) than we did (approximately 200-400 mg), and they used both vancomycin and cefuroxime as systemic 
perioperative prophylaxis. Of note, none of the 17 patients in our study who had received IV vancomycin for perioperative prophylaxis acquired an SSI. The results of the case series by Rasouli and Kopell and our retrospective cohort study suggest that a large prospective study should investigate the role of intrawound VP and perioperative vancomycin prophylaxis for the prevention of SSI after DBS placement. In addition, future studies should assess the dose of intrawound VP needed to prevent SSI.

The association between VP and decreased SSI risk did not quite achieve statistical significance (adjusted OR 0.32, 95\% CI 0.10-1.03, $\mathrm{p}=0.06$ ) when we controlled for patient sex. This result may suggest that the "protective" effect of male sex is driving the differences in SSI seen with the use of VP; however, males were not significantly overrepresented among the patients receiving VP (approximately $67 \%$ male) as compared with the patients not receiving VP (approximately 65\% male). Instead, the lack of statistical significance after calculating an adjusted OR may be related to a lack of power in our study. Given that the incidence of SSI is relatively low, the ability to detect a further reduction in the incidence with VP would require a larger number of patients than is reported on here.

We found that the duration of surveillance substantially affected the number of SSIs identified and, thus, the SSI rate. When we applied the new NHSN surveillance duration of 90 days for procedures involving implants, our SSI rate following DBS implantation was $3.7 \%$ compared with $6.5 \%$ when we applied the prior surveillance duration of 1 year. Published SSI rates after DBS procedures have ranged from $0 \%$ to $23 \%$. . $^{7-11,16,20,21,25,28,41,42,46,50}$ Differences in the duration of surveillance probably account for some of this variation in published SSI rates. Our findings suggest that truncating SSI surveillance at 90 days after DBS procedures may result in an underestimation of the true burden of SSI.

Consistent with the published literature, ${ }^{16,21,25}$ grampositive bacteria were the most common microorganisms isolated from SSIs in our study population. In fact, $75 \%$ of the infections were caused by methicillin-susceptible $S$. aureus (MSSA), P. acnes, or both. Recent observational studies have found that $P$. acnes SSI tends to have an indolent course and presents late. ${ }^{27,41} \mathrm{We}$ found similar results; however, we also found that SSIs caused by more virulent organisms occurred later, on average, than those caused by less virulent organisms. Thus, one cannot predict which organism is causing an infection based on the time to infection.

Our findings have clinical implications as well. Given that approximately $44 \%$ of the SSIs occurred more than 90 days after the procedures and that approximately $38 \%$ were caused by skin commensals alone, clinicians must be aware that infectious complications of DBS implantation can occur well outside the immediate postoperative period and that relatively avirulent organisms can cause SSI in this patient population. Longer-term infectious monitoring is warranted for these patients. Our data do not particularly inform causative differences between early and late SSI. More specifically and as seen in Table 1, virulent pathogens (for example, Pseudomonas sp., Serratia sp., MSSA, Enterobacter $\mathrm{sp}$.) did manifest SSIs both early and late (> 90 days). Additional studies with larger numbers of both
SSIs and patients are needed to identify initial procedurerelated factors (that is, contamination) versus potential seeding of DBS implants from unrelated sites (that is, urinary tract infection, pneumonia, bacteremia).

Several studies have not found a relationship between SSI risk and patient demographics ${ }^{7,11,45}$ or between SSI and the reason for placing the DBS..$^{454}$ In contrast, Bhatia et al. found that comorbidities were more frequent among patients with SSI than among those without SSI. ${ }^{7}$ Thus, we were surprised that the ASA score, a proxy measure for patient comorbidities, was not associated with SSI risk in our study. Of the patient-related and procedure-related factors we analyzed, only female sex was associated with SSI. Other groups have found procedure-specific differences in SSI rates between men and women. ${ }^{15,35}$ For example, a very large German study found that men had a higher SSI rate after abdominal operations than did women, although men had a lower SSI rate after cardiac operations. ${ }^{35}$ These investigators also found that the microorganisms isolated from the SSI varied by patient sex, with coagulase-negative staphylococci being isolated more commonly from SSIs in men, and MSSA and Pseudomonas aeruginosa being isolated more frequently from SSIs in women. Sex steroids modulate epidermal and dermal thickness and, thus, could affect wound healing. ${ }^{17}$ Moreover, results of a recent study of the skin microbiota indicate that skin flora varies by sex, which may help to explain the sex difference we found.$^{54}$

We did not find associations between SSI risk and procedure-related factors such as procedure duration. This observation is consistent with the results of previously published studies ${ }^{11,25,45,49}$ and suggests that interventions targeting specific aspects of the procedure itself may not decrease the SSI rate significantly.

Our study is one of the few to compare SSI rates after staged and unstaged DBS implantation. Some groups have reported higher SSI rates after staged procedures, ${ }^{16}$ whereas others have found no difference. ${ }^{49}$ We found that the SSI risk did not differ between patients who underwent staged procedures and those who underwent unstaged procedures. But because very few patients in our study had unstaged DBS implantations, our finding could have resulted from a type II error.

All patients who acquired SSIs after DBS implantation were readmitted to the hospital, and all required additional surgery, some even requiring multiple surgeries. Thus, these infections increase patient suffering, morbidity, and costs. In particular, patients whose DBS systems are removed are likely to have their motor or psychiatric symptoms recur, ${ }^{19}$ which is particularly debilitating for patients who depend on their DBS for symptom control. For example, the wife of a patient whose DBS system was infected recently told one of the authors (L.A.H.), "without the DBS there is no life."

To reduce the burden caused by SSI after DBS implantation, we must identify and implement effective preventive measures. Our data did not reveal any modifiable patient- or procedure-related factors associated with a lower SSI rate, though we and others have yet to examine other factors that may impact the SSI rate, such as preoperative warming and intraoperative body temperature or the 
number of individuals participating in the surgery. Given that we did not identify either patient-related or procedurerelated factors that could be addressed to decrease the risk of SSI, our observation that intrawound VP was associated with a lower risk of SSI than that in our standard practice may be very important.

This study has several strengths. Although retrospective cohort studies can be subject to selection bias and recall bias, we attempted to minimize these potential biases by collecting objective data from the medical records of all patients who had primary DBS implantations performed by one surgeon during the study period. We also attempted to minimize undocumented inconsistencies in procedural technique by restricting the study population to those patients whose procedures were performed by a single neurosurgeon at a single institution. In addition, an experienced infection preventionist independently conducted SSI surveillance.

This study also has some limitations. First, we were limited to the information included in the patient medical records. Second, a small proportion of patients underwent unstaged DBS placement, and these patients may not be representative of those undergoing unstaged DBS placement. Therefore, data about patient- and procedure-related factors associated with unstaged procedures must be interpreted with caution. Third, although our study period was longer than 10 years, the number of patients with SSIs was relatively low. Thus, the confidence intervals for the association between intrawound VP and SSI crossed 1 . Fourth, the effect of VP use could be confounded by other variables such as surgeon experience or by additive or synergistic effects with preoperative antibiotics. The impact of surgeon experience is likely to be minimal as the use of VP was initiated 7 years into the senior surgeon's practice. Additive or synergistic effects with topical VP would probably have manifested as covariance in the data; however, no relationship was identified between perioperative antibiotics and SSI incidence. Finally, as this study was based on procedures performed by a single surgeon at a single institution, the results may not be generalizable to all patients undergoing DBS implantation.

\section{Conclusions}

This retrospective cohort study is, to our knowledge, the first to compare SSI rates after DBS procedures between patients who did and those who did not receive VP. We found that the risk of SSI was $68 \%$ lower in the group that received intrawound VP and that intrawound VP was the factor most strongly associated with the risk of DBS hardware-related infections, although a sex difference weakened the statistical strength. This observation of potential VP efficacy is particularly important given the morbidity and costs associated with DBS-related infections and the paucity of other remediable patient-related and procedurerelated risk factors. Given the low baseline incidence of SSI after DBS implantation, a large prospective study is required to confirm our findings.

\section{Acknowledgments}

We thank Jean Pottinger, who did the surveillance for SSI, provided a list of patients who met the NHSN criteria for SSI, and conferred with investigators to ensure that patients were categorized correctly with respect to the presence or absence of an SSI.

\section{References}

1. Abdullah KG, Attiah MA, Olsen AS, Richardson A, Lucas TH: Reducing surgical site infections following craniotomy: examination of the use of topical vancomycin. J Neurosurg 123:1600-1604, 2015

2. Abode-Iyamah KO, Chiang HY, Winslow N, Park B, Zanaty $\mathrm{M}$, Dlouhy BJ, et al: Risk factors for surgical site infections and assessment of vancomycin powder as a preventive measure in patients undergoing first-time cranioplasty. J Neurosurg [epub ahead of print May 12, 2017; DOI: 10.3171/2016.12.JNS161967]

3. Bakhsheshian J, Dahdaleh NS, Lam SK, Savage JW, Smith ZA: The use of vancomycin powder in modern spine surgery: systematic review and meta-analysis of the clinical evidence. World Neurosurg 83:816-823, 2015

4. Bekhtereva NP, Grachev KV, Orlova AN: Iatsuksl. [Utilization of multiple electrodes implanted in the subcortical structure of the human brain for the treatment of hyperkinesis.] Zh Nevropatol Psikhiatr Im S S Korsakova 63:3-8, 1963 (Russian)

5. Benabid AL, Pollak P, Louveau A, Henry S, de Rougemont J: Combined (thalamotomy and stimulation) stereotactic surgery of the VIM thalamic nucleus for bilateral Parkinson disease. Appl Neurophysiol 50:344-346, 1987

6. Bhatia R, Dalton A, Richards M, Hopkins C, Aziz T, Nandi $\mathrm{D}$ : The incidence of deep brain stimulator hardware infection: the effect of change in antibiotic prophylaxis regimen and review of the literature. Br J Neurosurg 25:625-631, 2011

7. Bhatia S, Zhang K, Oh M, Angle C, Whiting D: Infections and hardware salvage after deep brain stimulation surgery: a single-center study and review of the literature. Stereotact Funct Neurosurg 88:147-155, 2010

8. Blomstedt P, Bjartmarz H: Intracerebral infections as a complication of deep brain stimulation. Stereotact Funct Neurosurg 90:92-96, 2012

9. Blomstedt P, Hariz MI: Hardware-related complications of deep brain stimulation: a ten year experience. Acta Neurochir (Wien) 147:1061-1064, 2005

10. Boviatsis EJ, Stavrinou LC, Themistocleous M, Kouyialis AT, Sakas DE: Surgical and hardware complications of deep brain stimulation. A seven-year experience and review of the literature. Acta Neurochir (Wien) 152:2053-2062, 2010

11. Brandmeir N, Nehrbass E, McInerney J: An analysis of scalp thickness and other novel risk factors for deep brain stimulator infections. Cureus 8:e792, 2016

12. Bratzler DW, Dellinger EP, Olsen KM, Perl TM, Auwaerter PG, Bolon MK, et al: Clinical practice guidelines for antimicrobial prophylaxis in surgery. Surg Infect (Larchmt) 14:73-156, 2013

13. Centers for Disease Control and Prevention: The National Healthcare Safety Network (NHSN) Manual: Procedureassociated Module: Division Surgical Site Infection (SSI). (http://www.cdc.gov/nhsn/pdfs/pscmanual/9pscssicurrent. pdf) [Accessed November 17, 2017]

14. Chiang HY, Herwaldt LA, Blevins AE, Cho E, Schweizer ML: Effectiveness of local vancomycin powder to decrease surgical site infections: a meta-analysis. Spine J 14:397-407, 2014

15. Chopra A, Klassen BT, Stead M: Current clinical application of deep-brain stimulation for essential tremor. Neuropsychiatr Dis Treat 9:1859-1865, 2013 
16. Constantoyannis C, Berk C, Honey CR, Mendez I, Brownstone RM: Reducing hardware-related complications of deep brain stimulation. Can J Neurol Sci 32:194-200, 2005

17. Dao H Jr, Kazin RA: Gender differences in skin: a review of the literature. Gend Med 4:308-328, 2007

18. Deuschl G, Schade-Brittinger C, Krack P, Volkmann J, Schäfer H, Bötzel K, et al: A randomized trial of deep-brain stimulation for Parkinson's disease. N Engl J Med 355:896908, 2006

19. Dlouhy BJ, Reddy A, Dahdaleh NS, Greenlee JD: Antibiotic impregnated catheter coverage of deep brain stimulation leads facilitates lead preservation after hardware infection. J Clin Neurosci 19:1369-1375, 2012

20. Doshi PK: Long-term surgical and hardware-related complications of deep brain stimulation. Stereotact Funct Neurosurg 89:89-95, 2011

21. Fenoy AJ, Simpson RK Jr: Risks of common complications in deep brain stimulation surgery: management and avoidance. J Neurosurg 120:132-139, 2014

22. Ghobrial GM, Cadotte DW, Williams K Jr, Fehlings MG, Harrop JS: Complications from the use of intrawound vancomycin in lumbar spinal surgery: a systematic review. Neurosurg Focus 39(4):E11, 2015

23. Gionfriddo MR, Greenberg AJ, Wahegaonkar AL, Lee KH: Pathways of translation: deep brain stimulation. Clin Transl Sci 6:497-501, 2013

24. Godil SS, Parker SL, O’Neill KR, Devin CJ, McGirt MJ: Comparative effectiveness and cost-benefit analysis of local application of vancomycin powder in posterior spinal fusion for spine trauma: clinical article. J Neurosurg Spine 19:331335,2013

25. Gorgulho A, Juillard C, Uslan DZ, Tajik K, Aurasteh P, Behnke E, et al: Infection following deep brain stimulator implantation performed in the conventional versus magnetic resonance imaging-equipped operating room. J Neurosurg 110:239-246, 2009

26. Greenberg BD, Askland KD, Carpenter LL: The evolution of deep brain stimulation for neuropsychiatric disorders. Front Biosci 13:4638-4648, 2008

27. Haleem A, Chiang HY, Vodela R, Behan A, Pottinger JM, Smucker J, et al: Risk factors for surgical site infections following adult spine operations. Infect Control Hosp Epidemiol 37:1458-1467, 2016

28. Halpern CH, Mitchell GW, Paul A, Kramer DR, McGill KR, Buonacuore D, et al: Self-administered preoperative antiseptic wash to prevent postoperative infection after deep brain stimulation. Am J Infect Control 40:431-433, 2012

29. Horan TC, Gaynes RP, Martone WJ, Jarvis WR, Emori TG: CDC definitions of nosocomial surgical site infections, 1992: a modification of CDC definitions of surgical wound infections. Infect Control Hosp Epidemiol 13:606-608, 1992

30. Johnson MD, Miocinovic S, McIntyre CC, Vitek JL: Mechanisms and targets of deep brain stimulation in movement disorders. Neurotherapeutics 5:294-308, 2008

31. Khan NR, Thompson CJ, DeCuypere M, Angotti JM, Kalobwe E, Muhlbauer MS, et al: A meta-analysis of spinal surgical site infection and vancomycin powder. J Neurosurg Spine 21:974-983, 2014

32. Krack P, Batir A, Van Blercom N, Chabardes S, Fraix V, Ardouin $\mathrm{C}$, et al: Five-year follow-up of bilateral stimulation of the subthalamic nucleus in advanced Parkinson's disease. $\mathbf{N}$ Engl J Med 349:1925-1934, 2003

33. Kuhn J, Lenartz D, Huff W, Lee S, Koulousakis A, Klosterkoetter J, et al: Remission of alcohol dependency following deep brain stimulation of the nucleus accumbens: valuable therapeutic implications? J Neurol Neurosurg Psychiatry 78:1152-1153, 2007

34. Levy D, Shabat-Simon M, Shalev U, Barnea-Ygael N, Cooper
A, Zangen A: Repeated electrical stimulation of reward-related brain regions affects cocaine but not "natural" reinforcement. J Neurosci 27:14179-14189, 2007

35. Lyons MK: Deep brain stimulation: current and future clinical applications. Mayo Clin Proc 86:662-672, 2011

36. Mayberg HS, Lozano AM, Voon V, McNeely HE, Seminowicz D, Hamani C, et al: Deep brain stimulation for treatmentresistant depression. Neuron 45:651-660, 2005

37. Miller JP, Acar F, Burchiel KJ: Significant reduction in stereotactic and functional neurosurgical hardware infection after local neomycin/polymyxin application. J Neurosurg 110:247-250, 2009

38. Müller UJ, Sturm V, Voges J, Heinze HJ, Galazky I, Heldmann M, et al: Successful treatment of chronic resistant alcoholism by deep brain stimulation of nucleus accumbens: first experience with three cases. Pharmacopsychiatry 42:288-291, 2009

39. Nuttin B, Cosyns P, Demeulemeester H, Gybels J, Meyerson B: Electrical stimulation in anterior limbs of internal capsules in patients with obsessive-compulsive disorder. Lancet 354:1526, 1999

40. O'Neill KR, Smith JG, Abtahi AM, Archer KR, Spengler DM, McGirt MJ, et al: Reduced surgical site infections in patients undergoing posterior spinal stabilization of traumatic injuries using vancomycin powder. Spine J 11:641-646, 2011

41. Pepper J, Zrinzo L, Mirza B, Foltynie T, Limousin P, Hariz $\mathrm{M}$ : The risk of hardware infection in deep brain stimulation surgery is greater at impulse generator replacement than at the primary procedure. Stereotact Funct Neurosurg 91:5665, 2013

42. Piacentino M, Pilleri M, Bartolomei L: Hardware-related infections after deep brain stimulation surgery: review of incidence, severity and management in 212 single-center procedures in the first year after implantation. Acta Neurochir (Wien) 153:2337-2341, 2011

43. Rasouli JJ, Kopell BH: The adjunctive use of vancomycin powder appears safe and may reduce the incidence of surgical-site infections after deep brain stimulation surgery. World Neurosurg 95:9-13, 2016

44. Rehncrona S, Johnels B, Widner H, Törnqvist AL, Hariz M, Sydow O: Long-term efficacy of thalamic deep brain stimulation for tremor: double-blind assessments. Mov Disord 18:163-170, 2003

45. Rodriguez-Oroz MC, Obeso JA, Lang AE, Houeto JL, Pollak P, Rehncrona S, et al: Bilateral deep brain stimulation in Parkinson's disease: a multicentre study with 4 years follow-up. Brain 128:2240-2249, 2005

46. Sillay KA, Larson PS, Starr PA: Deep brain stimulator hardware-related infections: incidence and management in a large series. Neurosurgery 62:360-367, 2008

47. Strom RG, Pacione D, Kalhorn SP, Frempong-Boadu AK: Decreased risk of wound infection after posterior cervical fusion with routine local application of vancomycin powder. Spine (Phila Pa 1976) 38:991-994, 2013

48. Sun B, Liu W: Stereotactic surgery for eating disorders. Surg Neurol Int 4 (Suppl 3):S164-S169, 2013

49. Sweet FA, Roh M, Sliva C: Intrawound application of vancomycin for prophylaxis in instrumented thoracolumbar fusions: efficacy, drug levels, and patient outcomes. Spine (Phila Pa 1976) 36:2084-2088, 2011

50. Tolleson C, Stroh J, Ehrenfeld J, Neimat J, Konrad P, Phibbs F: The factors involved in deep brain stimulation infection: a large case series. Stereotact Funct Neurosurg 92:227-233, 2014

51. Vandewalle V, van der Linden C, Groenewegen HJ, Caemaert J: Stereotactic treatment of Gilles de la Tourette syndrome by high frequency stimulation of thalamus. Lancet 353:724, 1999

52. Wichmann T, DeLong MR: Deep brain stimulation for move- 
ment disorders of basal ganglia origin: restoring function or functionality? Neurotherapeutics 13:264-283, 2016

53. Xiong L, Pan Q, Jin G, Xu Y, Hirche C: Topical intrawound application of vancomycin powder in addition to intravenous administration of antibiotics: a meta-analysis on the deep infection after spinal surgeries. Orthop Traumatol Surg Res 100:785-789, 2014

54. Ying S, Zeng DN, Chi L, Tan Y, Galzote C, Cardona C, et al: The influence of age and gender on skin-associated microbial communities in urban and rural human populations. PLoS One 10:e0141842, 2015

\section{Disclosures}

The authors report no conflict of interest concerning the materi- als or methods used in this study or the findings specified in this paper.

\section{Author Contributions}

Conception and design: Greenlee, Herwaldt. Acquisition of data: Greenlee, Abode-Iyamah, Woodroffe, Park, Nagahama, Winslow. Analysis and interpretation of data: all authors. Drafting the article: Greenlee, Abode-Iyamah, Chiang, Jareczek, Nagahama, Herwaldt. Critically revising the article: Greenlee, Abode-Iyamah, Chiang, Jareczek, Nagahama, Herwaldt. Reviewed submitted version of manuscript: Abode-Iyamah, Chiang, Jareczek, Herwaldt. Approved the final version of the manuscript on behalf of all authors: Greenlee. Statistical analysis: Chiang, Herwaldt. Study supervision: Greenlee.

\section{Correspondence}

Jeremy D. W. Greenlee: University of Iowa Hospitals and Clinics, Iowa City, IA. jeremy-greenlee@uiowa.edu. 